\title{
Magnetotransport properties of NiFe-Ag granular alloys: Origin of the thermal behavior
}

\author{
F. Badia, X. Batlle, ${ }^{\text {a) }}$ and A. Labarta ${ }^{\text {b) }}$ \\ Departament Física Fonamental, Universitat de Barcelona, Diagonal 647, 08028 Barcelona, \\ Catalonia, Spain \\ M. L. Watson \\ Centre for Data Storage Materials, Coventry University, Priory Street, Coventry CVI 5FB, United Kingdom
}

A. B. Johnston and J. N. Chapman

Department of Physics and Astronomy, University of Glasgow, Glasgow G12 8QQ, United Kingdom

(Received 11 October 1996; accepted for publication 11 April 1997)

\begin{abstract}
The effect of the temperature and magnetic field on the giant magnetoresistivity (GMR) of two FeNi-Ag granular alloys of composition $\mathrm{Fe}_{11.4} \mathrm{Ni}_{6.4} \mathrm{Ag}_{82.2}$ and $\mathrm{Fe}_{7.6} \mathrm{Ni}_{16.4} \mathrm{Ag}_{76.0}$ is discussed. Both samples were prepared by rf magnetron sputtering. Parts of them were rapidly annealed at 600, 650, and $750{ }^{\circ} \mathrm{C}$. All samples displayed giant magnetoresistivity which decays from its maximum value with a $T^{m}$ behavior, with $m \approx 0.8-0.9$, suggesting that the decrease in the maximum magnetoresistivity is due to the reduction in the particle magnetization associated with the spin wave excitation, which is a different mechanism to the electron-magnon interaction responsible for the $T$ dependence of GMR in magnetic multilayers. Magnetoresistivity $\rho_{M}$ decreases with temperature sharing essentially the same temperature decrease as the square of the macroscopic magnetization $M$ in the whole magnetic field range studied, which is due to the reduction in the particle magnetization and to superparamagnetic effects. The effect of the width of the particle size distribution and interparticle interactions on the linear relation $\rho_{M}$ vs $M^{2}$ are discussed. Care should be taken when representing $\rho_{M} / \rho(T, H=0)$ vs $\left(M / M_{s}\right)^{2}$ because the strong temperature-dependent slope shown in these plots is mainly due to the temperature dependence of both the resistivity $\rho(T, H=0)$ and $M_{s}$, and it is not an intrinsic $T$ dependence of GMR in granular alloys. Experimental results suggest that in granular materials, magnetoresistivity is dominated by magnetic moments at the surface of the particles, which also play a very important role in the demagnetization processes, and small magnetic particles. (C) 1997 American Institute of Physics.
\end{abstract}

[S0021-8979(97)00414-3]

\section{INTRODUCTION}

The recent discovery of giant magnetoresistance in $\mathrm{Fe} / \mathrm{Cr}$ magnetic multilayers ${ }^{1}$ has led to much current worldwide interest in the effect. This has, in part, been due to its potential exploitation by the data storage, magnetometry, and sensor industries. A number of theories have been developed which identify the large increase in the multilayer resistivity to be spin-dependent electron scattering. ${ }^{2}$ More recently, GMR has been found in heterogeneous alloys. ${ }^{3}$ These are formed by the codeposition of two immiscible metals, one of which is ferromagnetic in bulk. To promote phase segregation the film is either deposited at an elevated temperature, or annealed after deposition. This results in a distribution of fine ferromagnetic particles embedded in the nonmagnetic matrix material. In this case the GMR is due to the spindependent scattering of electrons either within or at the interfaces of the ferromagnetic particles dispersed throughout the nonmagnetic matrix. When the particle moments are randomly oriented, the resistivity of the material is higher than when they are aligned by the application of an external magnetic field. The effect has been observed in cosputtered thin films of many materials ${ }^{4}$ including $\mathrm{Co}-\mathrm{Ag}, \mathrm{Fe}-\mathrm{Ag}, \mathrm{NiFe}-$

\footnotetext{
${ }^{a)}$ Electronic mail: xavier@littlefly.ffn.ub.es

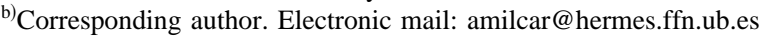

$\mathrm{Ag}, \mathrm{Co}-\mathrm{Au}, \mathrm{Ag}-\mathrm{Co}-\mathrm{Ni}$, and $\mathrm{Co}-\mathrm{Fe}-\mathrm{Ag}$, in melt spun ribbons of $\mathrm{Co}-\mathrm{Cu}^{5}$ and mechanically alloyed $\mathrm{Co}-\mathrm{Ag}{ }^{6}{ }^{6}$ The magnitude of the GMR which has been observed, has been found to be a sensitive function of both the size and the concentration of the ferromagnetic particles in the alloy. The former effect has been postulated to be due to the existence of an optimum particle size, determined by the conduction electron mean free path, ${ }^{7}$ (larger particle sizes result in a reduction of the GMR due to the decrease in particle surfaceto-volume ratio); and the latter effect to the onset of percolation, which acts to couple the particles ferromagnetically.

Concerning magnetic multilayers, when the electrical current is perpendicular to the multilayer plane (CPP-GMR), ${ }^{8,9}$ the spin diffusion length $l_{\text {sf }}$ acts as the key limiting parameter: $l_{\mathrm{sf}}$ is of the order of $10^{3} \AA$ (Ref. 10) and is therefore very much longer than the electron mean free path. It has been predicted that when $l_{\mathrm{sf}}$ is shorter than the film thicknesses, the CPP-GMR will decrease as exp $\left(-t_{N} / 2 l_{\mathrm{sf}}\right),{ }^{8,9} t_{N}$ being the thickness of the nonmagnetic layer, which has recently been reported for $\mathrm{Co} / \mathrm{Ag}$ multilayers (see references in Ref. 9). Due to the granular nature of heterogeneous alloy films, the electron transport processes are similar to those which occur in the CPP-GMR and it is postulated that for small particles the dominant damping parameter will also be the spin diffusion length because a typi- 
cal granular structure contains magnetic particles of mean diameter from about 20 to $40 \AA$ which are separated in average within about $30-60 \AA$ (for example, for concentrations of magnetic components in $\mathrm{FeNi}-\mathrm{Ag}$ of about $20 \%) . l_{\mathrm{sf}}$ has been estimated to be at least $35 \AA$ in CoAg granular films ${ }^{11}$ and within 50-500 $\AA$ (depending on temperature and magnetic field) for particles of mean diameter of $15 \AA$ ( $\sigma$ $=0.2$ ), by numerical simulation. ${ }^{12}$ GMR has previously been modeled by considering the self-averaging of the electric field lines passing through the granular structure, ${ }^{13}$ and predicts that the GMR is quadratic in an applied field $H$. A second mode ${ }^{14}$ identifies both the spin dependent scattering potential and density of states of the $d$ band as being possible origins of the GMR. This model also predicts a quadratic $H$ dependence of the GMR, provided spin-flip scattering is negligible, and is supported by recent measurement of the magnetothermopower of Co-Ag granular alloys. ${ }^{15}$ More recently the temperature dependence of the GMR observed in granular alloys has been considered within the framework of a modified effective exchange interaction model. ${ }^{16}$ In this model the spin dependent scattering arises from the exchange interaction between conduction electrons and the magnetic scatterers and the thermal decrease of GMR is due to the reduction in the magnetization associated with the spin wave excitation.

In order to elucidate the electron scattering mechanisms which influence the GMR in granular materials, we have investigated the temperature dependence of the resistivity and GMR of several FeNi-Ag alloys, as have been previously done in multilayers. ${ }^{17}$ Previous results for the present alloys may be found in Ref. 18 .

\section{EXPERIMENT}

$\mathrm{Ag}-\mathrm{Ni}-\mathrm{Fe}$ films of thickness $200-300 \mathrm{~nm}$ were rf sputtered onto glass microscope slides using a Nordiko 2000 sputtering system. The base pressure was less than 2 $\times 10^{-7}$ Torr, the sputtering pressure was 8 mTorr of argon and the sputtering power was $300 \mathrm{~W}$. The target used consisted of a 4 in. $\mathrm{Ag}(99.999 \%)$ disc onto which were placed $\mathrm{Ni}_{80} \mathrm{Fe}_{20}$ and $\mathrm{Fe} 0.25 \mathrm{~cm}^{2}$ squares arranged in a mosaic pattern. The film thickness was measured using a Tolansky multiple beam interferometer and the composition determined using energy dispersive $\mathrm{x}$-ray microanalysis (EDX) on a Philips electron microscope. As the sputtering rate from $\mathrm{Ni}_{80} \mathrm{Fe}_{20}$ and $\mathrm{Fe}$ are very similar, the film composition could be easily controlled by adding or removing squares of either of the two magnetic materials. In all cases the $\mathrm{Ag}$ content of the films was fixed at $70-80$ at. \%. To promote postdeposition phase segregation and magnetic particle growth, strips 7 $\mathrm{mm}$ wide diced from the substrate were rapidly annealed in a vacuum system with $2 \mathrm{~kW}$ halogen bulbs which produce a power density at the substrate of $80 \mathrm{~W} / \mathrm{cm}^{2}$. The bulb illumination is computer controlled and the temperature of the strips measured using a thermocouple mounted directly onto the sample holder. A number of annealing strategies were investigated varying from short thermal pulses $\left(750^{\circ} \mathrm{C}\right.$ attained in $40 \mathrm{~s})$ to moderately long anneals $\left(450{ }^{\circ} \mathrm{C}\right.$ for 10 $\min )$.
A number of thin films with various compositions were studied by using a Philips XRD system. Two films of composition $\mathrm{Fe}_{4.4} \mathrm{Ni}_{14.2} \mathrm{Ag}_{81.5}$ (sample D1) and $\mathrm{Fe}_{3.8} \mathrm{Ni}_{13.6} \mathrm{Ag}_{82.6}$ (sample $\mathrm{H1}$ ) deposited onto $\mathrm{SiN}$ windowed $\mathrm{Si}_{3} \mathrm{~N}_{4}$ substrates were investigated using transmission electron microscopy by bright field imaging and selected area diffraction using a Philips CM20 and EDX using a VG HB5.

Magnetic and transport properties were measured on films which had composition $\mathrm{Fe}_{11.4} \mathrm{Ni}_{6.4} \mathrm{Ag}_{82.2}$ (sample A) and $\mathrm{Fe}_{7.6} \mathrm{Ni}_{16.4} \mathrm{Ag}_{76.0}$ (sample $\mathrm{B}$ ). Three annealing temperatures were investigated: 600,650 , and $750{ }^{\circ} \mathrm{C}$, and these were reached in $20 \mathrm{~s}, 2 \mathrm{~min}$, and $3 \mathrm{~min}$, respectively. We will refer to them as $\mathrm{A}($ as-cast), $\mathrm{A}(600), \mathrm{A}(650), \mathrm{A}(750), \mathrm{B}$ (ascast), $B(600), B(650)$, and $B(750)$, respectively.

Resistivity and magnetoresistivity of all A and B samples were measured by an ac four point probe technique in the temperature range $20-300 \mathrm{~K}$ (in a closed cycle He gas cryostat) and in $H$ up to $12 \mathrm{kOe}$. The relative geometry of the film plane, the electrical current and the magnetic field was set by three ways: (a) the electrical current and $H$ are parallel to the film plane (parallel geometry); (b) the in-plane magnetic field is perpendicular to the electrical current (transverse geometry); and (c) $H$ is perpendicular to both the electrical current and the film plane (perpendicular geometry).

The zero-field-cooled (ZFC) and field-cooled (FC) processes at low fields and the magnetization curves were carried out in the temperature range $4.2-300 \mathrm{~K}$ with a SQUID magnetometer with the magnetic field applied along the film plane.

\section{RESULTS}

\section{A. X-ray diffraction and transmission electron microscopy}

$\mathrm{X}$-ray diffraction shows that the as-deposited films consist of a highly $\langle 111\rangle$ textured structure of $\mathrm{Ni}, \mathrm{Fe}$, and $\mathrm{Ag}$. The position of the main diffraction peak which can be attributed to the Ag matrix was shifted slightly from that of pure Ag. This effect has been seen previously, ${ }^{4}$ being associated with the incorporation of $\mathrm{Ni}$ and $\mathrm{Fe}$ atoms in the $\mathrm{Ag}$ matrix. On annealing, a very small shoulder appears on the side of the diffraction pattern, which indicates the phase separation of the ferromagnetic materials from the $\mathrm{Ag}$ matrix. These features have previously been seen in $\mathrm{NiFe}-\mathrm{Ag}$ alloys and in $\mathrm{CoFe}-\mathrm{Ag}$ alloys. ${ }^{4}$

Electron diffraction patterns (Fig. 1) from samples D1 and $\mathrm{H} 1$ confirmed the fcc structure. The as-deposited film (D1) shows diffuse diffraction rings whereas in the annealed film (H1) the rings are better defined, consistent with improvement of the long range order. The texturing is present in both samples and can be seen from the nonuniform intensity distribution around individual diffraction rings when the samples are tilted. This is especially marked in the $\{200\}$ and $\{220\}$ rings. The bright field TEM images (Fig. 2) show crystallites with a wide distribution of sizes, predominantly in the range $5-20 \mathrm{~nm}$. The crystallites in $\mathrm{H} 1$ have more clearly defined grain boundaries, consistent with the annealing treatment to which that film has been subjected. 

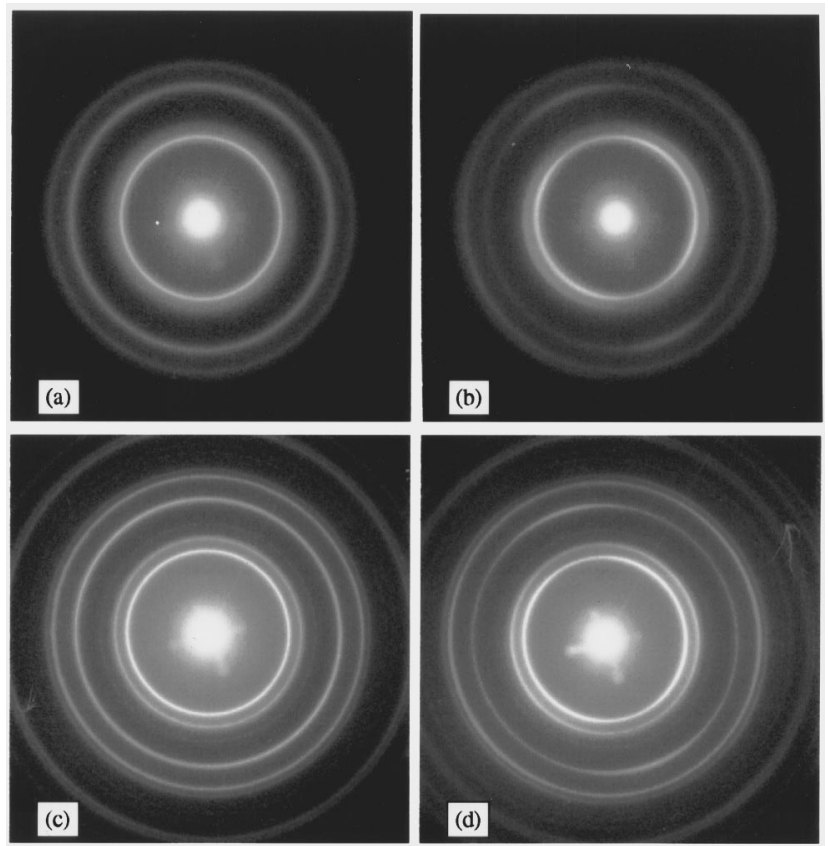

FIG. 1. Electron diffraction patterns of sample D1 (as-deposited) at a tilt of (a) $0^{\circ}$ and (b) $30^{\circ}$, and of sample $\mathrm{H} 1$ (annealed at $450^{\circ} \mathrm{C}$ for $10 \mathrm{~min}$ ), at a tilt of (c) $0^{\circ}$ and (d) $30^{\circ}$

Figures 3(a) and 3(c) show the annular dark field (ADF), and Figs. 3(b) and 3(d) the high-angle annular dark field (HADF) images of the specimen. These images were observed using electrons which have been scattered away from the direction of the incident beam, where the HADF images were obtained using only those electrons which were scattered through relative large angles (typically $>70 \mathrm{mrad}$ ). The ADF images show predominantly the crystallite structure, being complementary to the bright field images of Fig. 2.
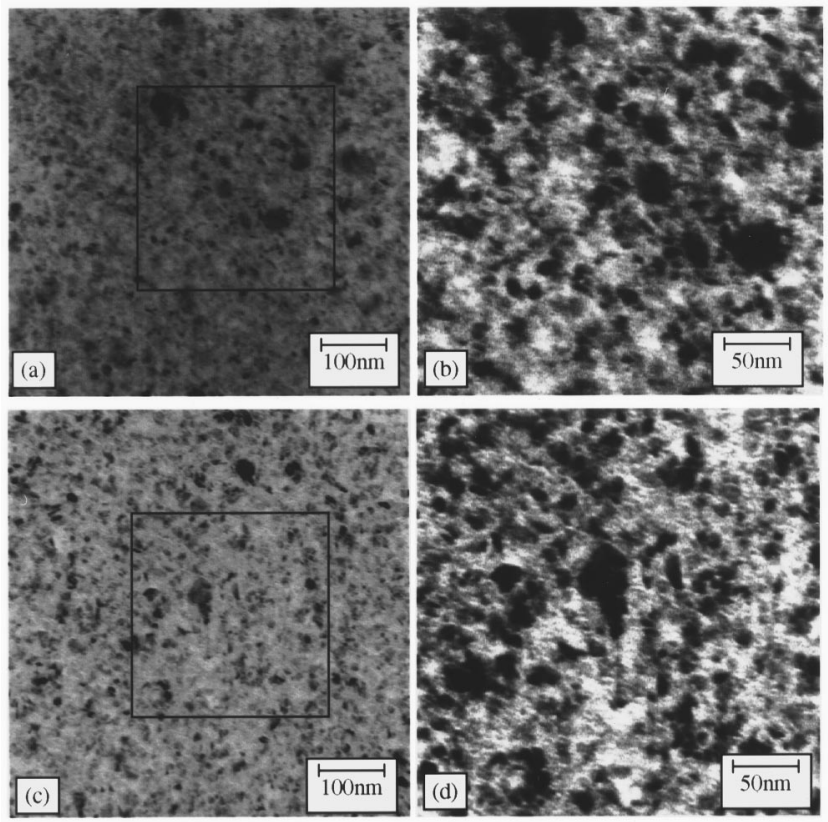

FIG. 2. Bright field TEM images for (a) sample D1 (as-deposited) and (c) $\mathrm{H} 1$ (annealed at $450{ }^{\circ} \mathrm{C}$ for $10 \mathrm{~min}$ ). (b) and (d) are enlarged insets of the areas marked in (a) and (c), respectively.
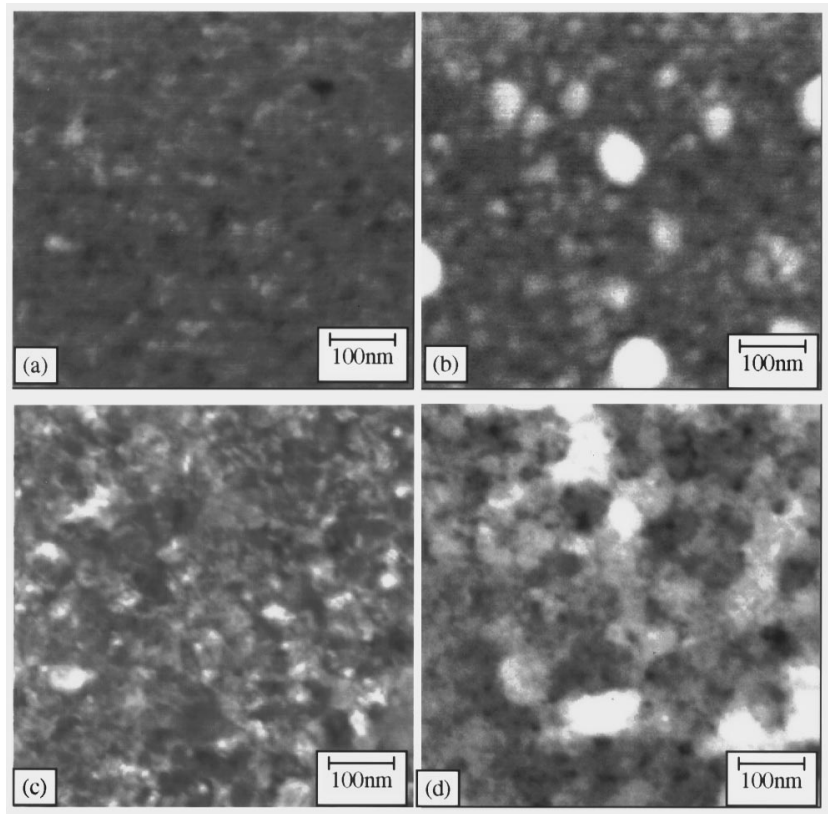

FIG. 3. Sample D1 (as-deposited): (a) ADF and (b) HADF images. Sample $\mathrm{H} 1$ (annealed at $450{ }^{\circ} \mathrm{C}$ for $10 \mathrm{~min}$ ): (c) $\mathrm{ADF}$ and (d) HADF images.

However, in the HADF images, the crystallographic contrast is substantially suppressed being strongly related to the local atomic number. ${ }^{19}$ Hence, for the systems under investigation here light regions are expected to be $\mathrm{Ag}$ rich and darker regions richer in FeNi. To obtain further information on the elemental distribution throughout the specimen, EDX analysis was used to obtain the compositional information given in Table I. X rays were detected while an electron probe of diameter $1.5 \mathrm{~nm}$ was rastered over areas of about 10 $\times 10 \mathrm{~nm}^{2}$. In each case, the probe was centered about a region that appeared light or dark in the HADF image. After completion of this procedure, a spectrum was recorded for a longer time period with the probe rastering over an area of $0.5 \times 0.5 \mu \mathrm{m}^{2}$ to determine the average film composition. The analyses themselves were based on the counts in the $K \alpha$ peaks of the elements of interest. Errors reflected the counting statistics and the count in the $\mathrm{Fe} K \alpha$ peak (the smallest of the three of interest) was typically 600 with an associated Poisson error of \pm 25 . The results in Table I show that there is a significant local compositional inhomogeneity in both samples as indicated by the HADF images and that

TABLE I. Compositions data from specimen (a) D1 and (b) H1.

\begin{tabular}{|c|c|c|c|}
\hline \multicolumn{4}{|c|}{ (a) D1 } \\
\hline & $\mathrm{Ag}$ & $\mathrm{Fe}$ & $\mathrm{Ni}$ \\
\hline Light & $87.6 \pm 1.4$ & $2.8 \pm 0.4$ & $9.6 \pm 1.2$ \\
\hline Dark & $78.9 \pm 1.7$ & $4.7 \pm 0.2$ & $16.4 \pm 1.5$ \\
\hline Matrix & $81.7 \pm 0.4$ & $4.1 \pm 0.2$ & $14.2 \pm 0.5$ \\
\hline \multicolumn{4}{|c|}{ (b) $\mathrm{H} 1$} \\
\hline & $\mathrm{Ag}$ & $\mathrm{Fe}$ & $\mathrm{Ni}$ \\
\hline Light & $91.0 \pm 0.9$ & $2.7 \pm 0.3$ & $6.3 \pm 1.0$ \\
\hline Dark & $66.6 \pm 13.5$ & $5.7 \pm 1.3$ & $27.8 \pm 13.2$ \\
\hline Matrix & $82.2 \pm 1.8$ & $3.8 \pm 0.7$ & $14.0 \pm 1.2$ \\
\hline
\end{tabular}



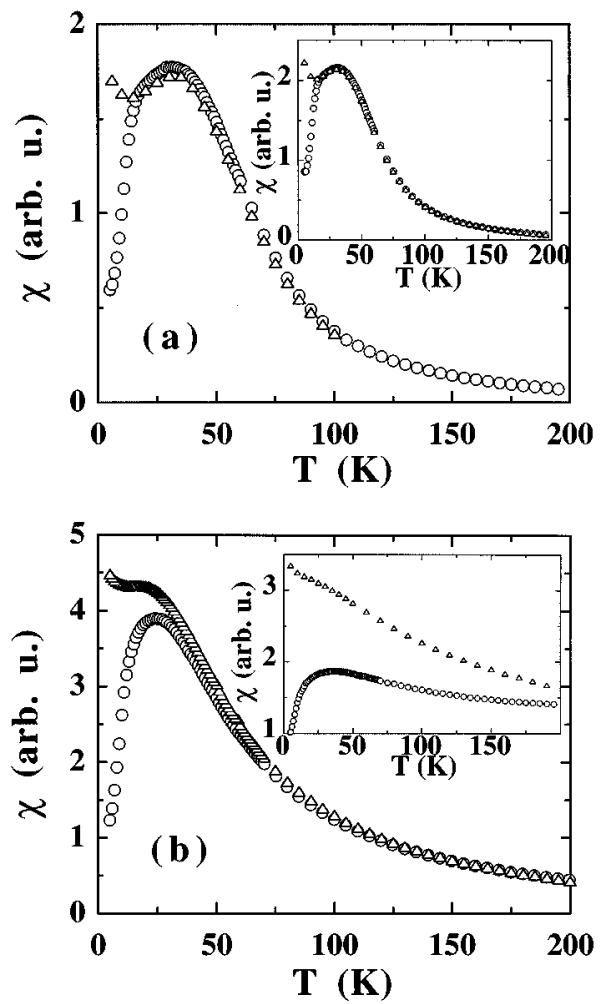

FIG. 4. (a) ZFC and FC processes measured at 100 Oe by applying the magnetic field along the film plane for sample A (as-cast). Inset: The same plot for sample A (600). (b) ZFC and FC processes measured at 100 Oe by applying the magnetic field along the film plane for sample A (650). Inset: The same for sample A (750).

the brighter regions are indeed rich in $\mathrm{Ag}$. However, the effect of the annealing is once again apparent there being a much wider spread in composition in H1 than in D1.

The mean-particle diameter, determined from fitting the room-temperature magnetization to a log-normal distribution of Langevin functions, was in the region of $10-30 \AA .{ }^{20}$ Therefore, the EDX data include a number of magnetic particles and matrix material, leading to the observed average composition. On annealing the films, TEM data, XRD, and low field susceptibility indicate that the particles grow and a granular character becomes prominent.

\section{B. Susceptibility measurements}

ZFC-FC processes for samples A(as-cast), A(600), $\mathrm{A}(650)$, and $\mathrm{A}(750)$ measured in $H=100$ Oe applied along the film plane are shown in Figs. 4(a) and 4(b). ZFC curves display a broad maximum at a temperature, $T_{M}(31.5,30.4$, 22.0 , and $36.7 \mathrm{~K}$ for $\mathrm{A}$ (as-cast), $\mathrm{A}(600), \mathrm{A}(650)$, and $\mathrm{A}(750)$, respectively). The maximum height at $T_{M}$ decreases and broadens with the annealing procedure, while the irreversibility (difference between the ZFC and the FC curves) grows progressively. These facts suggest that the particle size distribution broadens with increasing the annealing temperature, as shown by Greaves et al. ${ }^{21}$ although the domain formation of large ferromagnetic clusters with strong correlations may not be precluded. Those authors also found that the particle size distribution of as-cast samples showed some degree of bimodality which disappeared with annealing.
These may be related to the topological distribution of the ferromagnetic (FM) particles within the silver matrix: only FM particles surrounded by other FM particles are able to grow with annealing, while FM particles surrounded by silver particles do not grow, leading to a very broad particle size distribution and/or to large ferromagnetic interacting clusters, which lead to the persistence of the magnetic irreversibility up to high temperatures [Fig. 4(b) and inset]. We also note that magnetic irreversibility starts at a temperature well above the maximum of the ZFC curve for samples $A(650)$ and $A(750)$, while it starts at the peak for samples $\mathrm{A}$ (as-cast) and $\mathrm{A}(600)$, which might be tentatively attributed to the existence of magnetic particles that freeze at this temperature due to interparticle interactions. This freezing is also observed in the temperature dependence of the magnetoresistivity [see below, Fig. 9(a)], and it has already been reported in various granular films by Berkowitz et al. ${ }^{3}$ and Childress and Chien. ${ }^{22}$ The fact that the FC curves always increase below the ZFC maximum indicates the existence of very small superparamagnetic particles, although disordered surface spins might also contribute to the paramagnetic behavior. An order of magnitude of the mean magnetic particle diameter was obtained from the Curie-Weiss behavior followed by the ZFC-FC magnetization at high temperature, leading to $14 \AA$ for $\mathrm{A}($ as-cast) and $\mathrm{A}(600)$, and $21 \AA$ for $\mathrm{A}(650)$, suggesting that particles grow with annealing.

\section{Magnetoresistivity}

Let us define the magnetoresistivity $\left(\rho_{M}\right)$ as the difference

$$
\rho_{M}(T, H)=\rho(T, H=0)-\rho(T, H)
$$

where $\rho(T, H)$ is the resistivity measured at a temperature $T$ and in an applied magnetic field $H$. We assume that the total resistivity at $T$ and $H$ is given by ${ }^{17}$

$$
\rho(T, H)=\rho_{0}+\rho_{\mathrm{sd}}(T)+\rho_{M}(T, H),
$$

where: (i) $\rho_{0}$ is the resistivity due to defects, impurities and grain boundary scattering, and it is defined as the resistivity at $0 \mathrm{~K}$ and above the saturation field (magnetic field at which the resistivity no longer changes), in order to avoid any contribution arising from the misalignment of the magnetic moments, (ii) $\rho_{\mathrm{sd}}(T)$ is the temperature dependent resistivity due to the $s-d$ interband scattering mediated by phonons and magnons, and (iii) $\rho_{M}(T, H)$ is the magnetoresistivity as defined above.

Figures 5(a) and 5(b) show the temperature dependence of the magnetoresistivity change, defined as $-\rho_{M}(T, H) /$ $\rho(T, H=0)$, in the parallel geometry for samples $\mathrm{A}(650)$ and $B(650)$, respectively. All eight samples display very similar experimental features: (i) the resistivity decreases with increasing magnetic field as the magnetic moments become aligned along the field axis, (ii) the absolute value of the magnetoresistivity decreases with increasing temperature as particles become superparamagnetic and their magnetic moment is thermally randomized [Figs. 6(a) and 9], (iii) $\rho_{M}$ is not saturated at high fields at any temperature due to the existence of very small magnetic clusters that remain superparamagnetic, and (iv) the absolute value of the $\rho_{M}$ change 

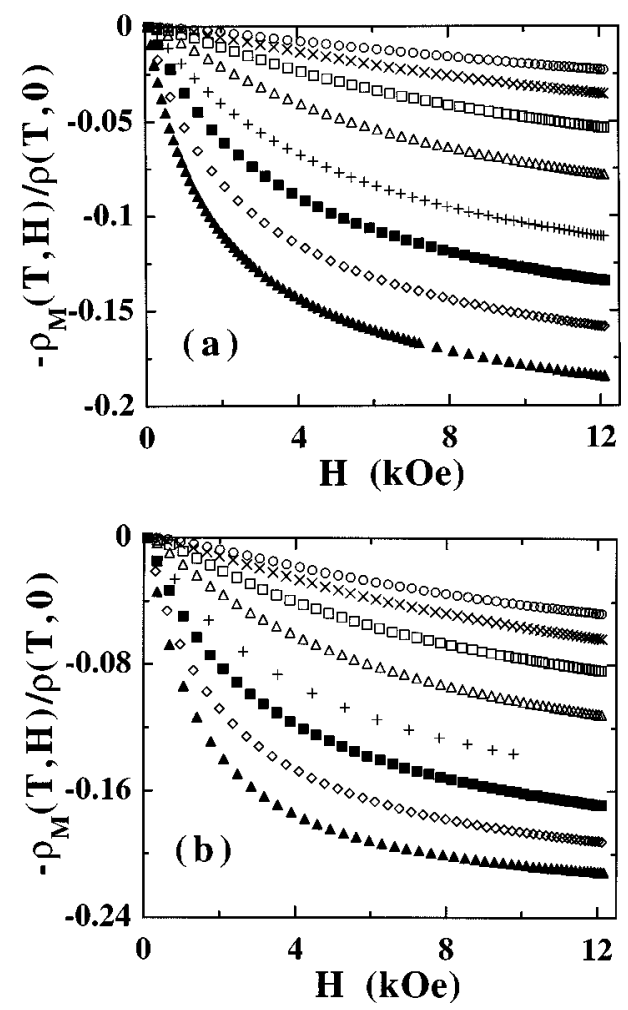

FIG. 5. (a) Magnetoresistivity change $-\rho_{M}(T, H) / \rho(T, H=0)$ as a function of $H$ at various temperatures in the parallel geometry for sample A (650). Temperatures: $(\boldsymbol{\Delta}) 21.5 \mathrm{~K}$; $(\diamond) 46.1 \mathrm{~K}$; $(\mathbf{\square}) 73.6 \mathrm{~K}$; (+) $102 \mathrm{~K}$; $(\triangle) 149.2$ $\mathrm{K}$; $(\square) 197.4 \mathrm{~K} ;(\times) 245 \mathrm{~K}$; (○) $290 \mathrm{~K}$. (b) Magnetoresistivity change $-\rho_{M}(T, H) / \rho(T, H=0)$ as a function of $H$ at different temperatures in the parallel geometry for sample B (650). Temperatures: $(\mathbf{\Lambda}) 21.5 \mathrm{~K}$; $(\diamond) 45.9$ $\mathrm{K} ;(\mathbf{\square}) 73.7 \mathrm{~K} ;(+) 102.4 \mathrm{~K} ;(\triangle) 149.6 \mathrm{~K} ;(\square) 197.8 \mathrm{~K} ;(\times) 245.9 \mathrm{~K} ;(\bigcirc)$ $282.1 \mathrm{~K}$.

depends on annealing temperature and there exists an optimum size and concentration for the ferromagnetic particles segregated in the silver matrix [Fig. 6(b)], as only scattering events within the spin diffusion length $\left(l_{\mathrm{sf}}\right)$ contribute to the resistivity (extra resistivity due to scattering from nonaligned ferromagnetic entities appears if the sizes and distances among them are smaller, or comparable, to $\left.l_{\mathrm{sf}}\right)$. From the fitting of the high-temperature magnetization curves [Fig. 11(a)] to a log-normal distribution of Langevin function the mean-magnetic diameter is found to be about $18 \AA(\sigma$ $=0.4)$ and $17 \AA(\sigma=0.6)$ for samples $\mathrm{A}(650)$ and $\mathrm{A}(750)$, respectively, in reasonable agreement with those found from the ZFC-FC curves. The value obtained for A(750) is only an order of magnitude since the superparamagnetic regime is not reached at $250 \mathrm{~K}$ [see inset of Fig. 4(b)]. From all these estimations an average interparticle distance of about $35 \AA$ is found, if we assume a regular array of spherical particles having a uniform diameter. This is only a rough estimation of the interparticle distance because the distribution of particle diameters is wide. We note that the mean-magnetic diameters are smaller than those crystalline diameters obtained from TEM (50-200 $\AA$ ), due to the fact that TEM does not detect the smallest particles and the existence of particle surface disordered spins. The average particle distances seem to be about the lowest limit for $l_{\text {sf }}{ }^{11,12}$ Also, the width of the distribution largely increases with the annealing at $750{ }^{\circ} \mathrm{C}$
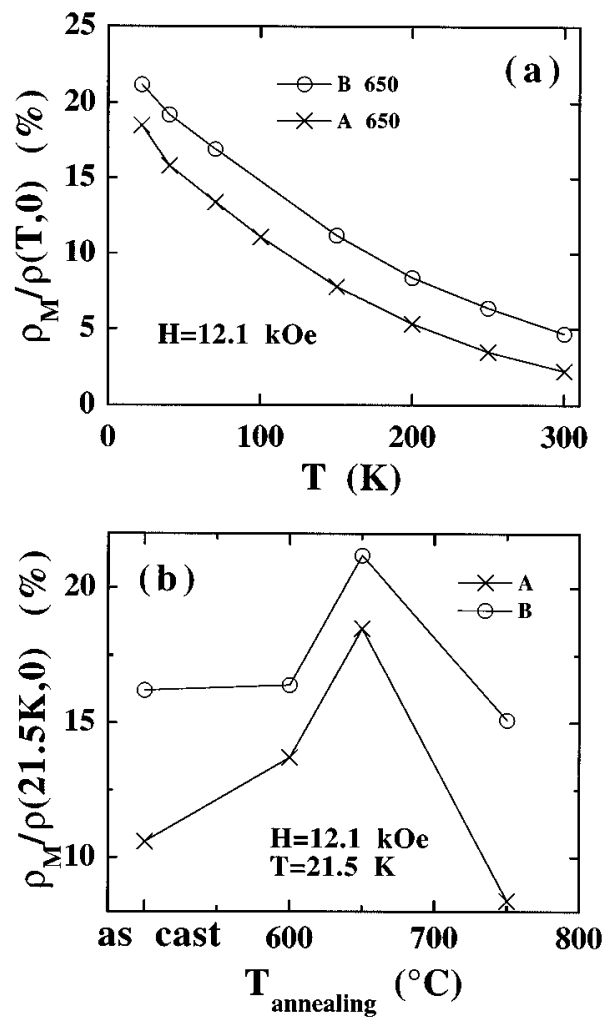

FIG. 6. (a) Absolute value of the maximum magnetoresistivity change at $12.1 \mathrm{kOe}, \rho_{M}(T, 12 \mathrm{kOe}) / \rho(T, H=0)$, as a function of temperature for samples $\mathrm{A}(650)(\times)$ and $\mathrm{B}(650)(\mathrm{O})$. (b) Absolute value of the maximum magnetoresistivity change at $12.1 \mathrm{kOe}$ and $21.5 \mathrm{~K}$, $\rho_{M}(21.5 \mathrm{~K}, 12 \mathrm{kOe}) / \rho(21.5 \mathrm{~K}, H=0)$, as a function of the annealing temperature $T_{a}$ for samples A $(\times)$ and $\mathrm{B}(\bigcirc)$.

(as also shown in ZFC-FC curves) which enhances the relative amount of the largest particles not contributing to the magnetoresistivity.

The residual-resistance-ratio (RRR) defined as $\mathrm{R}$ (highest $T, 12.1 \mathrm{kOe}$ )/R(lowest $T, 12.1 \mathrm{kOe}$ ) is given in Table II for all samples. RRR values are close to one and they do not change very much with particle size (they are all within 1.31.5 ), suggesting that the mean free path of the conduction electrons $(\lambda)$ is broadly constant with temperature and particle size distribution, indicating that impurity (defects) rather than phonon scattering is the dominant mechanism, although the latter is the responsible for the very small linear increase of the resistivity with temperature (Fig. 7). Let us then evaluate the main facts that account for the temperature dependence of the magnetoresistivity. We note that all magnetoresistivity measurements displayed in Figs. 5-10, 12, 14,

TABLE II. Residual-resistance-ratio (RRR), for samples A, $R(12.1 \mathrm{kOe}$, $290 \mathrm{~K}) / R(12.1 \mathrm{kOe}, 21.5 \mathrm{~K})$, and for samples $\mathrm{B}, R(12.1 \mathrm{kOe}, 282 \mathrm{~K}) /$ $R(12.1 \mathrm{KOe}, 21.5 \mathrm{~K})$

\begin{tabular}{lcc}
\hline \hline$T_{a}\left({ }^{\circ} \mathrm{C}\right)$ & Sample A & Sample B \\
\hline as-cast & 1.36 & 1.36 \\
600 & 1.40 & 1.35 \\
650 & 1.48 & 1.49 \\
750 & 1.50 & 1.32 \\
\hline \hline
\end{tabular}




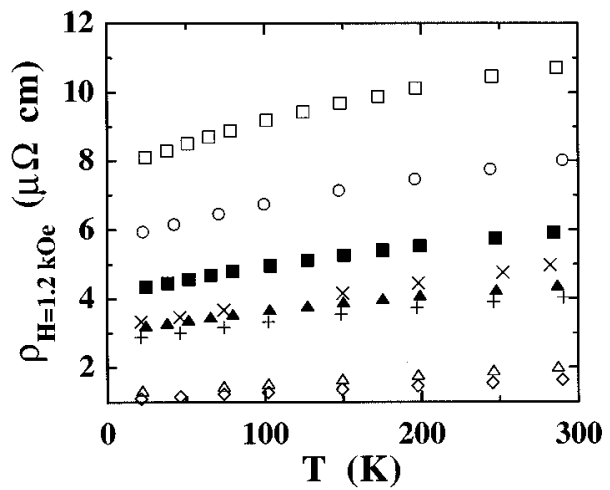

FIG. 7. Total resistivity $\rho(T, H)$ at $12.1 \mathrm{kOe}$ as a function of temperature for samples: $(\mathbf{\Delta}) \mathrm{A}($ as-cast), $(+) \mathrm{A}(600),(\diamond) \mathrm{A}(650),(\triangle) \mathrm{A}(750),(\boldsymbol{\square}) \mathrm{B}($ ascast), (○) B(600), (×) B(650), ( $\square) \mathrm{B}(750)$.

and 15 have been measured in decreasing field coming from the maximum experimental field available $(12.1 \mathrm{kOe})$.

The maximum $\rho_{M}$ changes are obtained for samples $\mathrm{B}(650)$ and $\mathrm{A}(650)$ (Fig. 6), tentatively suggesting that the optimum annealing temperature in these samples is about $650{ }^{\circ} \mathrm{C}$ in this $\mathrm{Ag}$ compositional range. $\rho_{M}$ changes are larger for samples B than for samples A which may be related to the larger amount of ferromagnetic entities within the silver matrix (see sample composition). However, the relative amount of $\mathrm{Fe}$ and $\mathrm{Ni}$ is also different between both samples and this could also contribute to the observed differences. GMR seems to be sharply reduced as the $\mathrm{Ni}: \mathrm{Fe}$ ratio is decreased. ${ }^{18}$

Concerning experimental geometries, $\rho_{M}$ is more susceptible to the magnetic field in the in-plane geometries than in the perpendicular (Fig. 8), which we assume is mainly a result of the interparticle dipolar interactions since (i) there is no difference between the $\rho_{M}$ change in the parallel and transverse geometry, and (ii) experimental magnetization curves can be reconstructed from magnetoresistivity measurements by assuming that a macroscopic (demagnetizing) dipolar field is responsible for the difference in field at which the $\rho_{M}$ is the same in the parallel and perpendicular geometries, as shown in Fig. 11(b) (see Sec. III D). Ferromagnetic resonance (FMR) data also showed clear angular dependence

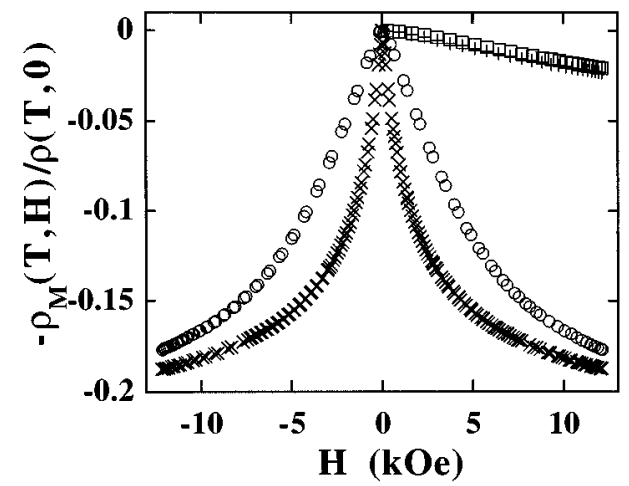

FIG. 8. Magnetoresistivity change $-\rho_{M}(T, H) / \rho(T, H=0)$ as a function of $H$ for sample $\mathrm{A}(650)$ at $21.5 \mathrm{~K}$ in the parallel geometry $(\times)$ and perpendicular geometry $(\bigcirc)$. The same plot at $290 \mathrm{~K}$ in the parallel geometry $(+)$ and perpendicular geometry $(\square)$.
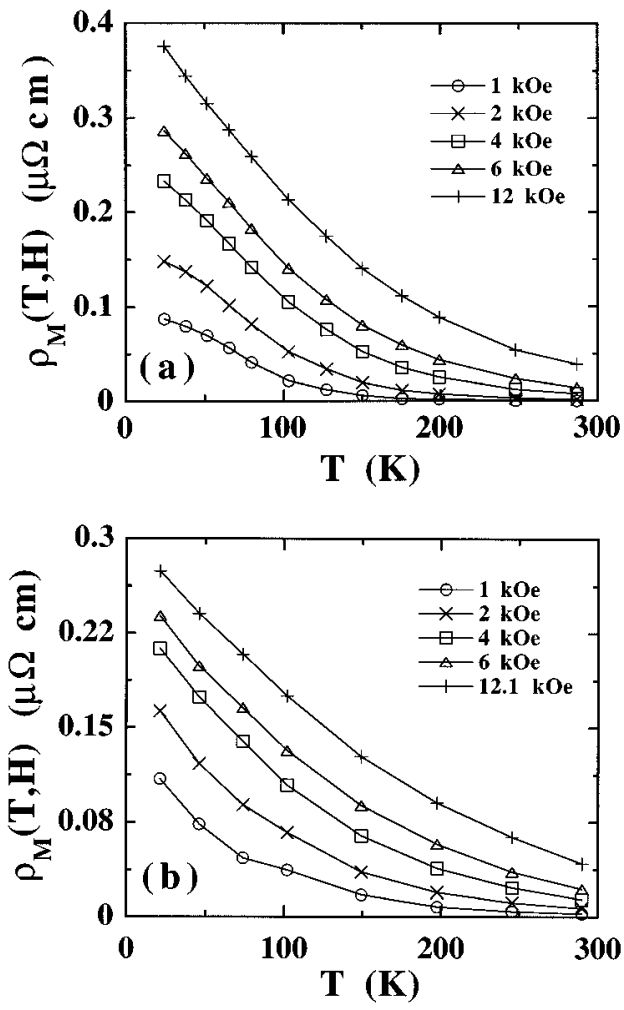

FIG. 9. $\rho_{M}(T, H)$ vs $T$ at various fields: (a) sample A(as-cast) and (b) sample $\mathrm{A}(650)$.

of the resonance frequency going from parallel to perpendicular geometry, ${ }^{23}$ which increases with the annealing temperature. This shape anisotropy may also be due to domain formation of large ferromagnetic clusters and their strong correlation. Besides, all measurements have been recorded with increasing and decreasing field, and we only observe some irreversibility below about $50 \mathrm{~K}$, which is associated with the hysteresis observed in the experimental magnetization curves [see the inset of Fig. 11(a)].

In Figs. 9(a) and 9(b) the temperature dependence of $\rho_{M}(T, H)$ at different fields for samples $\mathrm{A}$ (as-cast) and $\mathrm{A}(650)$, respectively, is shown. We note that the plot for

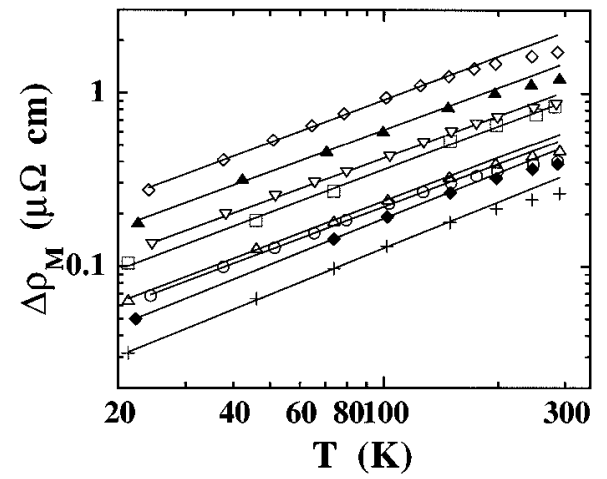

FIG. 10. Log-log plot of the temperature dependence of $\Delta \rho_{M}=\rho_{M}(T$ $=O, H)-\rho_{M}(T, H)$ at $12.1 \mathrm{kOe}$ for samples: $(\bigcirc) \mathrm{A}($ as-cast $),(\triangle) \mathrm{A}(600)$, $(+) \mathrm{A}(650),(\diamond) \mathrm{A}(750),(\boldsymbol{\Lambda}) \mathrm{B}($ as-cast $),(\square) \mathrm{B}(600),(\diamond) \mathrm{B}(650),(\nabla)$ $\mathrm{B}(750)$. Solid lines correspond to the best-fit of the data to a $T^{m}$ law. The $m$ exponents are given in Table III. 

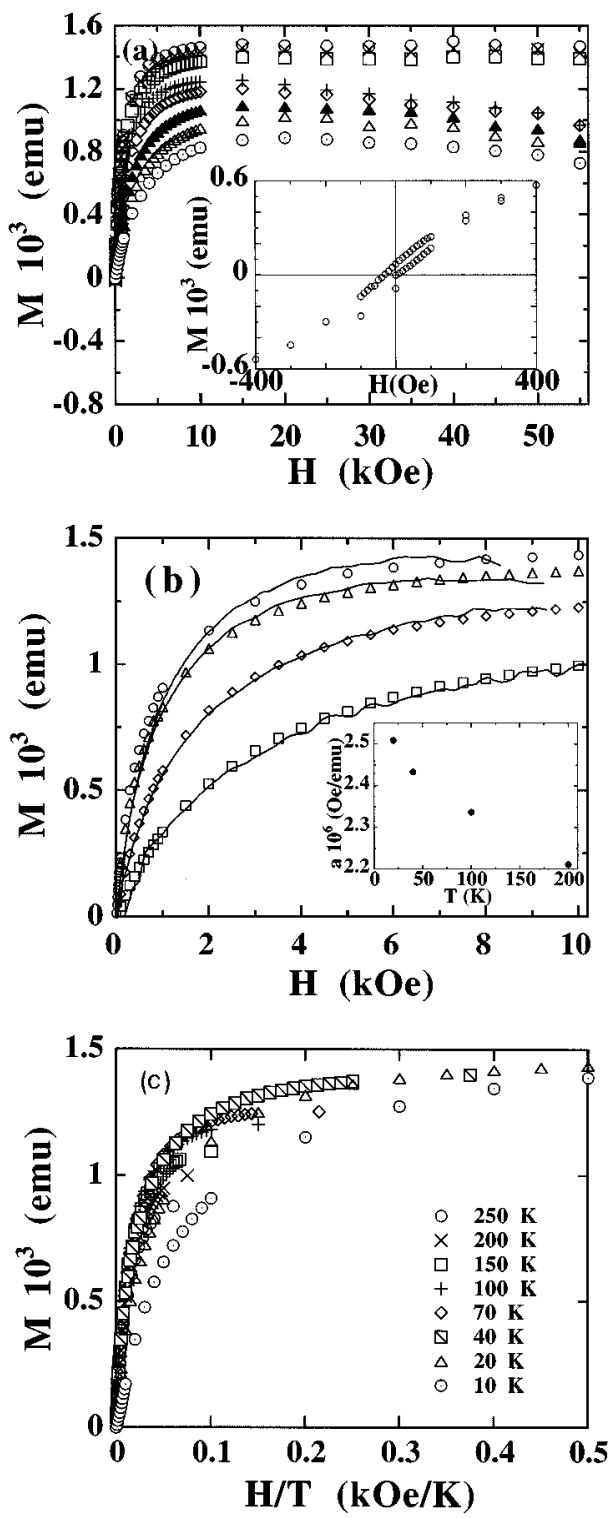

FIG. 11. (a) Experimental magnetization curves at various temperatures for sample A(650) at $(\bigcirc) 10 \mathrm{~K},(\times) 20 \mathrm{~K},(\square) 40 \mathrm{~K},(+) 70 \mathrm{~K},(\diamond) 100 \mathrm{~K},(\boldsymbol{\Delta})$ $150 \mathrm{~K},(\triangle) 200 \mathrm{~K}$, and $(\odot) 250 \mathrm{~K}$. Inset: Detail of the hysteresis cycle at 10 $\mathrm{K}$ for the same sample. (b) Macroscopic (demagnetizing) dipolar field $H_{D}$ (solid lines) obtained as described and compared to experimental magnetization curves at $(\bigcirc) 20 \mathrm{~K},(\triangle) 40 \mathrm{~K},(\diamond) 100 \mathrm{~K}$, and $(\square) 200 \mathrm{~K}$ for sample $\mathrm{A}(650)$. Inset: temperature dependence of $a_{\perp}(T)$. (c) Experimental magnetization curves plotted as a function of $H / T$ for sample $\mathrm{A}(650)$.

$\mathrm{A}(600)$ resembles the one for $\mathrm{A}$ (as-cast), and the plot for $\mathrm{A}(750)$ resembles the one for $\mathrm{A}(650) . \rho_{M}(T, H)$ displays a monotonic increase as the temperature goes down for samples $\mathrm{A}(650)$ and $\mathrm{A}(750)$, which is in agreement with the progressive blocking of the ferromagnetic particles [Fig. 4(b) and inset]. However, an inflection appears in the lowtemperature region at low fields for samples $\mathrm{A}$ (as-cast) and $\mathrm{A}(600)$, as found by Nigam et al. ${ }^{24}$ in $\mathrm{Au}_{87} \mathrm{Fe}_{13}$ cluster glass. A similar behavior is observed for B samples. This could indicate a degree of freezing due to interparticle interactions, as already reported from the $\mathrm{ZFC} / \mathrm{FC}$ curves in various granular films ${ }^{3,22}$ and as can be observed in Fig. 4(a) and inset. Interparticle interactions tend to flatten the FC magne-
TABLE III. Fitted $m$ exponents in the $\Delta \rho_{M}$ vs $T^{m}$ relationship for all A and B samples at $12.1 \mathrm{kOe}$.

\begin{tabular}{lcc}
\hline \hline & (a) & \\
$T_{a}\left({ }^{\circ} \mathrm{C}\right)$ & Sample A & Sample B \\
\hline as-cast & 0.82 & 0.81 \\
600 & 0.84 & 0.81 \\
650 & 0.89 & 0.83 \\
750 & 0.88 & 0.82 \\
& $(\mathrm{~b})$ & \\
$T_{a}\left({ }^{\circ} \mathrm{C}\right)$ & Sample A & Sample B \\
\hline as-cast & 0.77 & 0.93 \\
600 & 0.79 & 0.87 \\
650 & 0.88 & $\cdots$ \\
750 & 0.96 & 0.90 \\
\hline \hline
\end{tabular}

tization curve at temperatures below the ZFC maximum and consequently $\rho_{M}(T, H)$ also tends to flatten.

Let us define the reduction of $\rho_{M}$ at a finite temperature, $\rho_{M}(T, H)$, as referred to its value at $T=0, \rho_{M}(T=0, H)$ as

$$
\Delta \rho_{M}(T, H)=\rho_{M}(T=0, H)-\rho_{M}(T, H) .
$$

The log-log plot of $\Delta \rho_{M}$ vs $T$ is displayed in Fig. 10 for all A and B samples at $12.1 \mathrm{kOe} . \rho_{M}(T=0, H)$ has been obtained by extrapolating $\rho_{M}(T, H)$ at $T=0$. The slope of the plot yields the $m$ exponent in the relationship $\Delta \rho_{M} \approx T^{m}$ (see Table III), and this power law may give an idea of the underlying scattering mechanism. The $m$ exponent very slightly increases with annealing temperature [from 0.82 for $\mathrm{A}$ (ascast) to 0.88 for $\mathrm{A}(750)$, and from 0.81 for $\mathrm{B}$ (as-cast) to 0.82 for $\mathrm{B}(750)]$. Those values are far from the $m=1.5-2$ exponents found in magnetic multilayers, ${ }^{17,25}$ in which it has been shown that the electron-magnon scattering leads to these power laws and, consequently, the main mechanism responsible for the thermal decrease of $\Delta \rho_{M}$ is the spin mixing process associated with this interaction. On the contrary, our values are in close agreement with the $m=0.8$ exponent obtained by Wang and Xiao ${ }^{16}$ in granular $\mathrm{Co}_{20} \mathrm{Ag}_{80}$ thin film. As pointed out in Ref. 16, these low values seem to rule out the electron-magnon interaction as the main mechanism. These authors suggest that the spin-dependent scattering arises from an effective exchange interaction between conduction electrons and the magnetic scatterers. Then, $\Delta \rho_{M}$ decreases with temperature due to the reduction in the particle magnetization associated with the spin wave excitation, as shown in Sec. IV of this paper.

\section{Magnetization measurements}

We plot in Fig. 11(a) the experimental magnetization curves for $\mathrm{A}(650)$ between 10 and $250 \mathrm{~K}$ with the magnetic field applied along the film plane. A detail of the hysteresis cycle for $\mathrm{A}(650)$ at $10 \mathrm{~K}$ is displayed in the inset of Fig. 11(a), showing that the coercive field is small (about 150 Oe). Above about $10 \mathrm{kOe}$ the diamagnetic contribution of the substrate dominates magnetization curves.

The experimental magnetization curves can be reconstructed from magnetoresistivity measurements by assuming that dipolar interactions among the particles are responsible 
for the difference between the parallel and perpendicular magnetoresistivity (Fig. 8) leading to the appearance of a macroscopic demagnetizing field in the sample, $H_{D}$, which is proportional to the magnetization of the sample in each geometry. Assuming that the demagnetizing field in the parallel configuration is negligible, $H_{D}$ may be evaluated by the difference in field at which $\rho_{M}$ is the same in both geometries $\left(\rho_{M}^{\|}\right.$and $\left.\rho_{M}^{\perp}\right)$, that is

$$
H_{D}=H_{2}\left(\rho_{M}\right)-H_{1}\left(\rho_{M}\right)
$$

with $\rho_{M}=\rho_{M}^{\perp}\left(H_{2}, T\right)=\rho_{M}^{\|}\left(H_{1}, T\right)$. Note, that if $\rho_{M}$ is the same in both geometries the magnetization must also be the same $\left[M_{\perp}\left(H_{2}, T\right)=M_{\|}\left(H_{1}, T\right)\right]$. If we now plot $H_{D}$ as a function of $H_{1}$ or $H_{2}$ this should be proportional to the experimental magnetization in the parallel or perpendicular geometry, respectively, due to the fact that demagnetizing effects in the parallel geometry are negligible. In Fig. 11(b) the experimental parallel magnetization at four temperatures for sample $\mathrm{A}(650)$ is compared to $H_{D}\left(H_{1}\right)$ obtained as explained previously. To do both curves comparable in magnitude at each temperature, $H_{D}$ has to be divided by an arbitrary renormalization parameter, $a_{\perp}(T)$, which can be interpreted as the demagnetization factor in the perpendicular geometry. If magnetization were written in appropriate units and the sample were a uniform bidimensional ferromagnet, $a_{\perp}$ should be temperature independent and equal to $4 \pi$. The temperature dependence of $a_{\perp}(T)$ is shown in the inset of Fig. 11(b). $H_{D}\left(H_{1}\right)$ reproduces experimental $M_{\|}(H)$ quite correctly, which suggests that dipolar interactions among the particles are the major factor for the magnetoresistivity being anisotropic.

We note that $H_{D}$ is not due to the formation of a nonequilibrium pole density on the surface of the sample, since the samples are not uniform ferromagnetic films. $H_{D}$ is a net internal field that arises from interparticle dipolar interactions: the potential energy due to dipolar interactions is larger when the assembly of magnetic moments are aligned perpendicular to the plane of the film (magnetic field applied perpendicular to the film) than when they are aligned within the film plane (magnetic field applied parallel to the film plane). This fact indicates that a larger applied field is necessary to compensate dipolar interactions when the field is applied perpendicular than when it is applied parallel to the film plane and it explains magnetoresistivity being anisotropic. The demagnetization factor is temperature dependent since the interparticle dipolar interactions depend on the thermal dependence of the magnetization of all ferromagnetic particles in the sample (through the size distribution), as well as on their topological distribution.

We display in Fig. 11(c) the plot $M$ vs $H / T$ for sample $\mathrm{A}(650)$, which shows that experimental magnetization curves do not superimpose to give a single $H / T$ curve. Similar results are obtained for sample $\mathrm{A}(750)$. Although this may be taken as evidence of interparticle interactions, $M$ does not scale on $H / T$ if a broad distribution of particle volumes exists, as occurs in these samples.

\section{DISCUSSION}

In granular alloys with small single-domain particles, the external magnetic field does not change the size of the FM domains and it only rotates the magnetic axis of the particles, leading to a reduction of the resistance as the magnetic axes are progressively aligned. The magnetic moments of the grains are very large as compared to the moment of a single atom and thus we will apply classical theory to describe the system. If we assume that the field-dependent scattering mechanism is proportional to the degree of correlation of the moments of neighboring grains averaged over all configurations, $\left\langle\boldsymbol{\mu}_{i}(H) \cdot \boldsymbol{\mu}_{j}(H)\right\rangle / \mu^{2}$, where $\boldsymbol{\mu}_{i}$ and $\boldsymbol{\mu}_{j}$ are the magnetic moments of the $i$ th and $j$ th neighboring grains, with $\left|\boldsymbol{\mu}_{i}\right|=\left|\boldsymbol{\mu}_{j}\right|=\mu$, and ignoring scattering within the grains, the magnetoresistivity change with magnetic field may be expressed at a given temperature, as first shown by Gittleman, ${ }^{26}$ as

$$
\frac{\rho_{M}(T, H)}{\rho(T, H=0)} \propto \frac{\left\langle\boldsymbol{\mu}_{i}\left(H_{T}\right) \cdot \boldsymbol{\mu}_{j}\left(H_{T}\right)\right\rangle}{\mu^{2}}
$$

where $H_{T}$ is the total field that the particles experience, which, in the parallel geometry, is the vector sum of the applied field $H$, the anisotropy field and the interaction field (in principle, dipolar and exchange) arising from neighboring particles.

As $\left\langle\boldsymbol{\mu}_{i}\left(H_{T}\right) \cdot \boldsymbol{\mu}_{j}\left(H_{T}\right)\right\rangle / \mu^{2}=\left\langle\cos \phi_{i j}\left(H_{T}\right)\right\rangle$, the relevant fact in calculating the moment-moment correlation is the average value of $\cos \phi_{i j}\left(H_{T}\right)$ over the neighboring grains, being $\phi_{i j}$ the angle between the axes of FM entities. In order to correlate magnetoresistivity to the magnetization measurements, Eq. (5) is usually simplified by assuming that the anisotropy of the particles is random, particles are noninteracting and magnetic moments are uncorrelated, so $\left\langle\boldsymbol{\mu}_{i}\left(H_{T}\right)\right.$ - $\left.\boldsymbol{\mu}_{j}\left(H_{T}\right)\right\rangle / \mu^{2}=\left\langle\cos \theta_{i}\right\rangle^{2}=\left(M / M_{s}\right)^{2}$, where $\theta_{i}$ is the angle between the magnetic axis of the particle $i$ and the applied magnetic field, $M$ is the global magnetization, and $M_{s}$ is the saturation magnetization. Then, Eq. (5) becomes

$$
\frac{\rho_{M}(T, H)}{\rho(T, H=0)} \propto\left(\frac{M}{M_{S}}\right)^{2} .
$$

A deviation from Eq. (6) is expected in the case of a broad particle size distribution. Zhang and Levy ${ }^{7}$ predicted theoretically that deviation when considering a volume distribution of the type $f(V) \alpha 1 / V$ with $0 \leqslant V \leqslant V_{\max }$ and attributed it to the existence of a large number of very small particles, since they have a large relative contribution to the magnetoresistivity than to the magnetization. Bellouard et al. ${ }^{27}$ noted that such particle distribution is not realistic and attributed the increasing slope of magnetoresistivity with increasing $M^{2}$ in $\mathrm{Co}-\mathrm{Fe} / \mathrm{Ag}$ thin films to the alignment of the particle surface disordered spins under a high field, causing a large variation of the magnetoresistivity as compared to their contribution to the magnetization. However, Bellouard et al. ${ }^{27}$ pointed out that this surface spin disorder could also be interpreted as a size distribution effect: the outer shell of the particle will cause the decrease of the average magnetic moment of the particle with temperature and this effect may be viewed as a particle volume decrease with a constant particle magnetization, shifting the size distribution towardss- 


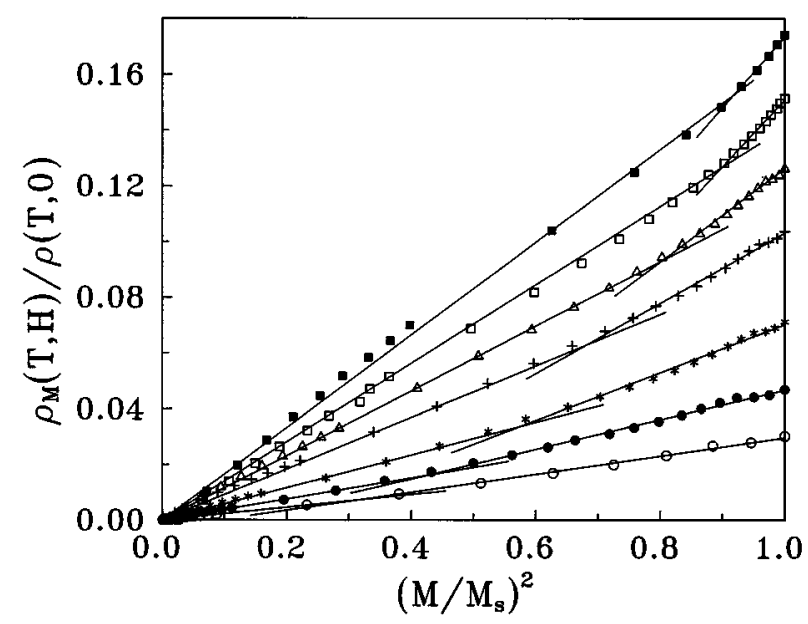

FIG. 12. $\rho_{M}(T, H) / \rho(T, H=0)$ as a function of $\left(M / M_{s}\right)^{2}$ for sample $\mathrm{A}(650)$ at $(\square) 20 \mathrm{~K},(\square) 40 \mathrm{~K},(\triangle) 70 \mathrm{~K},(+) 100 \mathrm{~K},(*) 150 \mathrm{~K},(\bullet) 200 \mathrm{~K}$, and $(\bigcirc) 250 \mathrm{~K}$. Solid lines indicate the linear relationship of the data in both field regimes $\left(H<H_{0}\right.$ and $\left.H>H_{0}\right)$.

mall particles and approaching the result from Zhang and Levy. ${ }^{7}$ For $\mathrm{FeNi}-\mathrm{Ag}$ granular alloys annealed at up to $750{ }^{\circ} \mathrm{C},{ }^{20}$ magnetization and magnetoresistivity at room temperature may be modeled using an ensemble of superparamagnetic particles which have a log-normal distribution of diameters. However, for the highest temperature annealing strategies, magnetoresistivity is accurately fit if only particles under a critical diameter of about $6 \mathrm{~nm}$ are considered.

Other physical mechanisms different from a broad size distribution may also be taken into account. Interparticle interactions will result in a deviation from Eq. (6) at low fields. ${ }^{28}$ Apart from this, $\rho_{M}(T, H) / \rho(T, H=0)$ cannot ever strictly follow the $\left(M / M_{s}\right)^{2}$ law since the magnetoresistivity depends on the short range order (e.g., on the local anisotropy field) while magnetization is an average over the whole system, as noted by El-Hilo et al., ${ }^{29}$ which leads to a clear experimental deviation from the quadratic law at low fields. Gehring et al. ${ }^{30}$ have recently proposed a new scattering mechanism: the conduction electron spin is rotated via a pseudo-Larmor precession induced by the internal exchange field of the magnetic particle. These authors showed that this mechanism acts to depolarize the conduction electron spins even when the magnetic moments of the particles are nearly parallel, leading to a relative increase at high fields of magnetoresistivity as compared to the magnetization.

We show in Fig. $12 \rho_{M}(T, H) / \rho(T, H=0)$ as a function of $\left(M / M_{s}\right)^{2}$ at various temperatures between 20 and $250 \mathrm{~K}$ for sample $\mathrm{A}(650)$. Magnetic field ranges from 0 to $10 \mathrm{kOe}$ and $M_{s}=M(10 \mathrm{kOe})$. Figure 12 evidences two different linear regimes, and that the value of $\left(M / M_{s}\right)^{2}$ at which the crossover from the first $\left[\mathrm{low}\left(M / M_{s}\right)^{2}\right.$ values] to the second $\left[\right.$ high $\left(M / M_{s}\right)^{2}$ values] regime takes place increases with decreasing temperature. The crossover $M / M_{s}$ values correspond to the following crossover applied magnetic fields $H_{0}$ : $(4.2 \pm 0.2) \mathrm{kOe}$ for sample $\mathrm{A}(650)$ from 70 to $250 \mathrm{~K}$ (increases to 5 and $7.9 \mathrm{kOe}$ at 40 and $20 \mathrm{~K}$, respectively) and (2.5 \pm 0.5$) \mathrm{kOe}$ for $\mathrm{A}(750)$ from 20 to $290 \mathrm{~K}$.

Then, the magnetoresistivity change as a function of the magnetization at a given temperature may be expressed as

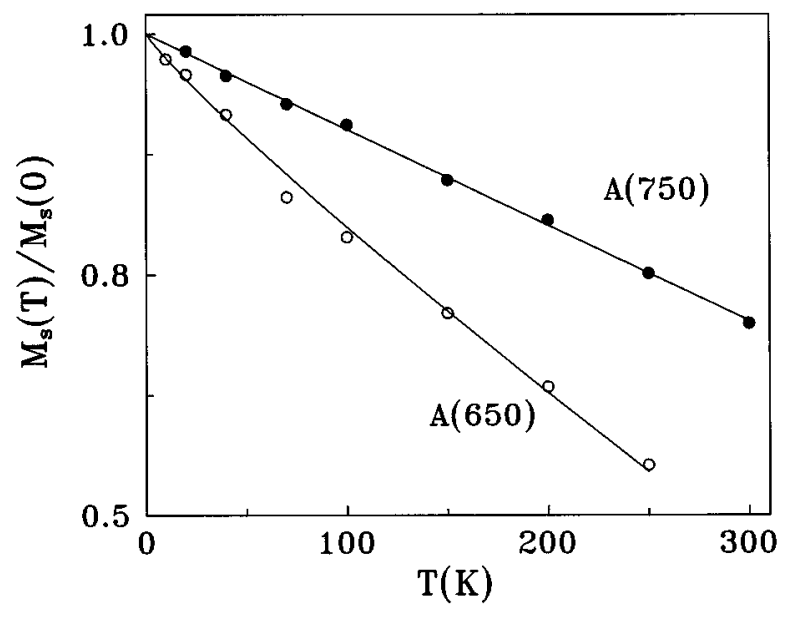

FIG. 13. Saturation magnetization $M_{s}=M(10 \mathrm{kOe})$ as a function of temperature for samples $\mathrm{A}(650)$ and $\mathrm{A}(750)$. Solid line represents the fit of data to a decay of the type $M_{s}(T)=M_{s}(T=0) \cdot\left(1-B \cdot T^{n}\right)$, with $n=0.9$ for $\mathrm{A}(650)$ and $n=1$ for $\mathrm{A}(750)$.

$$
\frac{\rho_{M}(T, H)}{\rho_{M}(T, 0)} \propto \alpha(H)\left(\frac{M}{M_{S}}\right)^{2} .
$$

If the existence of two linear regimes may be primarily attributed to the fact that the particle size distribution is broad, $\alpha(H)$ describes the reduction in the magnetoresistivity change [with respect to Eq. (6)] due to those particles which have a larger relative contribution to the magnetization than to the magnetoresistivity: at low fields $\left(H<H_{0}\right)$, the major contribution to the magnetization arises from the largest particles, which have a lower relative contribution to the magnetoresistivity change (low slope region in Fig. 12). When particle sizes are larger than $\lambda$, intraparticle scattering is the dominant mechanism, the system progressively behaves like a bulk ferromagnet and $\rho_{M}$ vanishes. On the other hand, at high fields $\left(H>H_{0}\right)$ the major contribution to the magnetization comes from the smallest particles (and from the spins at particle surface), which have a larger relative contribution to the magnetoresistivity change (high slope region in Fig. 12). Then, the two linear regimes may be explained assuming that (i) for $H>H_{0}, \alpha(H) \approx 1$ (all small particles contributing to magnetization also contribute to magnetoresistivity change) and (ii) for $H<H_{0}, \alpha(H) \approx \alpha_{0}<1$. If deviations are partially due to interparticle interactions and/or local anisotropy, at high fields magnetic particles follow the applied magnetic field if uncorrelated $[\alpha(H) \approx 1]$, while at low fields magnetic particles are less sensitive to the variation of the applied field $\left[\alpha(H) \approx \alpha_{0}<1\right]$. The crossover from the first to the second regime is characterized by a curvature in the relationship $\rho_{M}(T, H) / \rho(T, H=0)$ vs $\left(M / M_{s}\right)^{2}$.

Let us now consider the effect of temperature on the magnetoresistivity. As can be seen in Fig. 13, the saturation magnetization measured at $10 \mathrm{kOe}, M_{s}(T)$, for samples $\mathrm{A}(650)$ and $\mathrm{A}(750)$ decays from its maximum value, $M_{s}(0)$, following a law of the type $M_{s}(0) \cdot\left(1-B T^{n}\right)$, where $n$ is 0.9 and 1.0, respectively. The decay of $M_{s}(T)$ is faster in sample A(650) than in sample A(750) since magnetic particles are smaller in the former, which increases the relative contribution of the surface spins (surface spins de- 


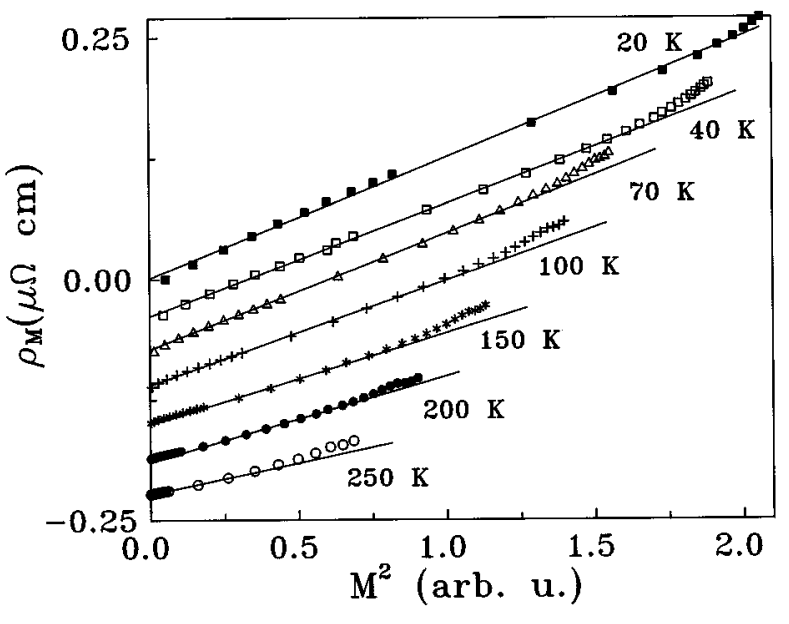

FIG. 14. Magnetoresistivity $\rho_{M}$ as a function of the square of the macroscopic magnetization $M$ up to $10 \mathrm{kOe}$ for sample $\mathrm{A}(650)$. Curves have been shifted arbitrarily along the vertical axis for clarity.

magnetize faster than bulk spins). Also, the small values of the $n$ exponent observed in both samples suggest that the spins at the particle surface play an important role in the demagnetization processes (saturation magnetization linearly decreases with temperature in 2D FM systems ${ }^{31}$ ). These values of the $n$ exponent are slightly larger than those found for the thermal decay of $\Delta \rho_{M}$ at high field, which suggest that $\Delta \rho_{M}$ shares the same thermal variation as the square of the saturation magnetization $M_{s}$. This fact can be justified taking into account that $\left[M_{s}(T) / M_{s}(0)\right]^{2}=1-2 B T^{n}+B^{2} T^{2 n}$ can be approximated by a law of the type $1-C T^{m}$, with $m$ slightly smaller than $n$, providing that $B \ll 1$, as is the case for samples $A(650)$ and $A(750)$. These phenomenological results indicate that $\Delta \rho_{M}$ at high fields decrease with temperature mainly due to the reduction in the particle saturation magnetization associated with the spin wave excitation.

It is then reasonable to assume that the magnetoresistivity $\rho_{M}$ at intermediate fields decreases with temperature essentially due to the reduction in the macroscopic magnetization associated with the reduction in the particle magnetization and superparamagnetic effects. This suggestion is confirmed in Fig. 14, where $\rho_{M}$ is plotted against $M^{2}$ for sample $\mathrm{A}(650)$ at several temperatures within 20 and $250 \mathrm{~K}$ (curves have been shifted arbitrarily along the vertical axis for clarity). All $\rho_{M}$ vs $M^{2}$ curves are essentially parallel in both field regimes (confirming the previous assumption) although their slopes slightly decrease with temperature, being the reduction is more important at high temperature, which could be attributed to the additional reduction in the magnetoresistivity associated with other spin mixing mechanisms, such as the electron-magnon interaction (the relative importance of this additional mechanism increases with temperature). The existence of these additional mechanisms is also confirmed by the deviation of $\Delta \rho_{M}$ from the expected $C T^{m}$ behavior at high temperatures as shown in Fig. 10. We note that the modified effective exchange interaction model ${ }^{16}$ takes into account the temperature decrease of $\rho_{M}(T, H) / \rho(T, H=0)$. However, it is thus relevant to point out that most of the thermal dependence of the slope in the linear relation $\rho_{M}(T, H) / \rho(T, H=0)$ vs $\left(M / M_{s}\right)^{2}$ (see Fig.

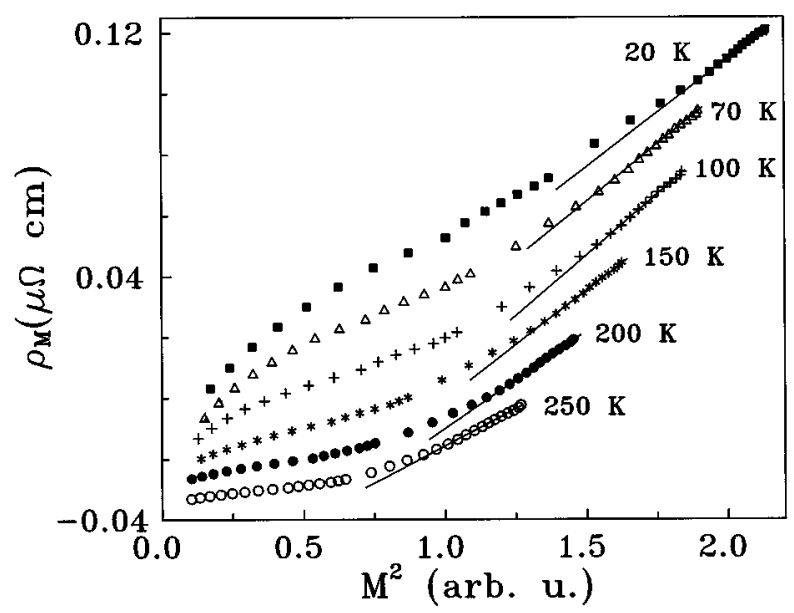

FIG. 15. The same plot that Fig. 14, for sample A(750).

12) is due to the thermal variation of both $\rho(T, H=0)$ and $M_{s}$, and it is not an intrinsic $T$ dependence of GMR in granular alloys. It can be shown that $M_{s}^{2} / \rho(T, H=0)$ reproduces the temperature decay of the slopes of $\rho_{M}(T, H) / \rho(T, H=0)$ vs $\left(M / M_{s}\right)^{2}$ in both field regimes (Fig. 12).

$\rho_{M}$ is plotted against $M^{2}$ for sample A(750) in Fig. 15 (curves have been shifted arbitrarily along the vertical axis for clarity). In the high field regime $\left(H>H_{0}\right)$, similar results than those obtained for sample $\mathrm{A}(650)$ are found and the relative temperature variation of the slope is exactly the same than in the latter. However, in the low field regime $(H$ $<H_{0}$ ) a more complex behavior is shown: the linear regime is observed only at high temperatures, while a clear deviation appears as temperature goes down. Similar results have been observed in $\mathrm{CoFe}-\mathrm{AgCu}$ granular alloys with high interparticle interactions. ${ }^{32}$ The ZFC-FC curves for sample $\mathrm{A}(750)$ indicate that the annealing procedure leads to a broadening of the particle size distribution and to the formation of large ferromagnetic clusters with strong magnetic correlations, thus suggesting that there are two contributions to the low field regime: (i) the first one coming from large isolated particles which are responsible for the initial part of the curves, and (ii) the second one coming from large ferromagnetic interacting clusters which are responsible for the relative decrease of the slope at intermediate fields, since a small increase in the magnetic field may cause an increase in the magnetization without modifying the relative orientation of the magnetic moments of the granules forming the clusters.

Let us then phenomenologically express the magnetoresistivity as:

$$
\begin{aligned}
& \frac{\rho_{M}(T, H)}{\rho(0,0)}=A \alpha_{0}\left(\frac{M(H)}{M_{s}(T=0)}\right)^{2} ; \quad H<H_{0} \\
& \begin{aligned}
\frac{\rho_{M}(T, H)-\rho_{M}\left(T, H_{0}\right)}{\rho(0,0)}= & A\left[\left(\frac{M(H)}{M_{s}(T=0)}\right)^{2}\right. \\
& \left.-\left(\frac{M\left(H_{0}\right)}{M_{s}(T=0)}\right)^{2}\right] ; \quad H>H_{0}
\end{aligned}
\end{aligned}
$$


where $A$ is a dimensionless parameter which is related to the spin dependent scattering efficiency of the particles. In Eq. (8) magnetoresistivity and magnetization have been arbitrarily normalized dividing them by the factors $\rho(0,0)$ and $M_{s}(T=0)$, respectively, in order to obtain a dimensionless relation. For sample $\mathrm{A}(650)$ the ratio of the slopes of both field regimes yields a temperature-independent $\alpha_{0}=0.7$, and the slope of the high field regime leads to an efficiency parameter $A=0.26$ up to $\sim 100 \mathrm{~K}$, which decreases down to $A=0.14$ at $250 \mathrm{~K}$. For sample $\mathrm{A}(750), A=0.11$ up to $200 \mathrm{~K}$ and decreases down to $A=0.08$ at $250 \mathrm{~K}$. As mentioned above, for this sample it is only possible to gain an estimation of $\alpha_{0}$ above $150 \mathrm{~K}$, leading to $\alpha_{0}=0.3$. We note that $A, \alpha_{0}$, and GMR [Fig. 6(b)] decrease with the annealing temperature since, as the width of the distribution increases $[\sigma=0.4$ for $\mathrm{A}(650)$ and $\sigma=0.6$ for $\mathrm{A}(750)]$, the relative amount of large particles not contributing to the magnetoresistivity change also increases.

In conclusion, experimental results show that the temperature dependence of the magnetoresistivity in $\mathrm{FeNi}-\mathrm{Ag}$ granular alloys follows essentially the temperature dependence of the square of the macroscopic magnetization, suggesting that the main mechanism responsible for the temperature decrease in GMR is the reduction in the macroscopic magnetization associated with both the reduction in the particle magnetization and superparamagnetic effects. However, other additional spin mixing mechanism should be taken into account at high temperature. Also, interparticle interactions arising from the microstructure of the sample effect the temperature dependence of GMR. Experimental results suggest that in granular materials, the magnetoresistivity is dominated by magnetic moments at the surface of the particles, which play a very important role in the demagnetization processes, and small magnetic particles.

\section{ACKNOWLEDGMENTS}

Financial support of both the Spanish CICYT through the MAT94-1024-CO2-02 Project and the Catalan CIRIT through the GRQ1012 Project (1993-96) are largely recognized. We would like to thank Professor J. M. González and C. De Julian from the Materials Science Institut CSICMadrid for recording some of the magnetization measurements. M.L.W. would like to acknowledge the support of Nuffield Foundation and EPSCR for funding his part of this work.

\footnotetext{
${ }^{1}$ M. N. Baibich, J. M. Broto, A. Fert, F. Nguyen Van Dau, F. Petroff, P. Eitanne, G. Creuzet, A. Friederich, and J. Chazelas, Phys. Rev. Lett. 61, 2472 (1988).

${ }^{2}$ R. E. Camley and J. Barnas, Phys. Rev. Lett. 63, 664 (1989); A. Barthelemy and A. Fert, Phys. Rev. B 43, 13124 (1991); B. Dieny, Europhys. Lett. 17, 261 (1992); P. M. Levy, S. Zhang, and A. Fert, Phys. Rev. Lett. 65, 1643 (1990); S. Zhang, P. M. Levy, and A. Fert, Phys. Rev. B 45, 8689 (1992).
}

${ }^{3}$ A. E. Berkowitz, J. R. Mitchell, M. J. Carey, A. P. Young, S. Zhang, F. E. Spada, F. T. Parker, H. Hutten, and G. Thomas, Phys. Rev. Lett. 68, 3745 (1992); J. Q. Xiao, J. S. Jiang, and C. L. Chien, Phys. Rev. Lett. 68, 3749 (1992).

${ }^{4}$ M. J. Carey, A. P. Young, A. Starr, D. Rao, and A. E. Berkowitz, Appl. Phys. Lett. 61, 2935 (1992); A. Tsoukatos, H. Wan, G. C. Hadjipanayis, K. M. Unruh, and Z. G. Li, J. Appl. Phys. 73, 5509 (1993); J. S. Jiang, J. Q. Xiao, and C. L. Chien, Appl. Phys. Lett. 61, 2362 (1992); M. Kitada, K. Yamamoto, and N. Shimizu, J. Magn. Magn. Mater. 124, 243 (1993).

${ }^{5}$ J. Wecker, R. von Helmolt, L. Schultz, and K. Samwer, Appl. Phys. Lett. 62, 1985 (1993)

${ }^{6}$ S. M. Thompson, J. F. Gregg, C. R. Staddon, D. Daniel, S. J. Dawson, K. Ounadjela, J. Hammann, C. Fermon, G. Saux, K. O'Grady, S. J. Grieves, J. M. Coey, and A. Fagan, Philos. Mag. B 68, 923 (1994).

${ }^{7}$ S. Zhang, Appl. Phys. Lett. 61, 1855 (1992); S. Zhang and P. M. Levy, J. Appl. Phys. 73, 5315 (1993); P. Xiong, G. Xiao, J.-Q. Wang, J. Q. Xiao, J. S. Jiang, and C. L. Chien, Phys. Rev. Lett. 69, 3220 (1992).

${ }^{8}$ M. Johnson, Phys. Rev. Lett. 67, 3594 (1991).

${ }^{9}$ T. Valet and A. Fert, Phys. Rev. B 48, 7099 (1993).

${ }^{10}$ W. P. Pratt, Jr., S.-F. Lee, J. M. Slaughter, R. Loloee, P. A. Schroeder, and J. Bass, Phys. Rev. Lett. 66, 3060 (1991); S.-F. Lee, W. P. Pratt, Jr., R. Loloee, P. A. Schroeder, and J. M. Bass, Phys. Rev. B 46, 548 (1992).

${ }^{11}$ M. B. Stearns and Y. Cheng, J. Appl. Phys. 75, 6894 (1994).

${ }^{12}$ J. F. Gregg, W. Allen, S. M. Thompson, M. L. Watson, and G. A. Gehring, J. Appl. Phys. 79, 5593 (1996)

${ }^{13}$ S. Zhang, Appl. Phys. Lett. 61, 1855 (1992).

${ }^{14}$ L. Xing and Y. C. Chang, Phys. Rev. B 48, 4156 (1993); L. Xing, Y. C. Chang, M. B. Salamon, D. M. Frankel, J. Shi, and J. P. Lu, Phys. Rev. B 48, 6728 (1993)

${ }^{15}$ L. Piraux, M. Cassart, J. S. Jiang, J. Q. Xiao, and C. L. Chien, Phys. Rev. B 48, 638 (1993); J. Shi, E. Kita, L. Xing, and M. B. Salamon, Phys. Rev. B 48, 16119 (1993).

${ }^{16}$ J.-Q. Wang and G. Xiao, Phys. Rev. B 50, 3423 (1994); J.-Q. Wang, P. Xiong, and G. Xiao, Phys. Rev. B 47, 8341 (1993); J.-Q. Wang and G. Xiao, Phys. Rev. B 49, 3982 (1994).

${ }^{17}$ J. E. Mattson, M. E. Brubaker, C. H. Sowers, M. Conover, Z. Qin, and S. D. Bader, Phys. Rev. B 44, 9378 (1991).

${ }^{18}$ M. L. Watson, K. O’Grady, and V. G. Lewis, J. Appl. Phys. 75, 6927 (1994).

${ }^{19}$ S. J. Pennycook, S. D. Berger, and R. J. Culberston, J. Microsc. 144, 229 (1986).

${ }^{20}$ J. Wiggins, M. L. Watson, P. A. Gago-Sandoval, X. Batlle, F. Badia, and A. Labarta, J. Appl. Phys. 79, 5605 (1996).

${ }^{21}$ S. J. Greaves, M. El-Hilo, and K. O'Grady, J. Appl. Phys. 76, 6802 (1994).

${ }^{22}$ J. R. Childress and C. L. Chien, Phys. Rev. B 43, 8089 (1991).

${ }^{23}$ M. L. Watson (private communication).

${ }^{24}$ A. K. Nigam, S. Radha, and G. Chandra, J. Phys., Condens. Matter. 5, 9197 (1993)

${ }^{25}$ M. A. M. Gijs, S. K. J. Lenczowski, and J. B. Giesbers, Phys. Rev. Lett. 70, 3343 (1993)

${ }^{26}$ J. I. Gittleman, Y. Goldstein, and S. Bozowski, Phys. Rev. B 5, 3609 (1972); J. I. Gittleman, B. Abeles, and S. Bozowski, Phys. Rev. B 9, 3891 (1974)

${ }^{27}$ C. Bellouard, B. George, and G. Marchal, J. Phys., Condens. Matter. 6, 7239 (1994)

${ }^{28}$ J. F. Gregg, S. M. Thompson, S. J. Dawson, K. Ounadjela, C. R. Staddon, J. Hamman, C. Ferman, and K. O’Grady, Phys. Rev. B 49, 1064 (1994).

${ }^{29}$ M. El-Hilo, K. O'Grady, and R. Chantrell, J. Appl. Phys. 76, 6811 (1994).

${ }^{30}$ G. A. Gehring, J. F. Gregg, S. M. Thompson, and M. L. Watson, J. Magn. Magn. Mater. 140-144, 501 (1995).

${ }^{31}$ G. Xiao and C. L. Chien, J. Appl. Phys. 61, 4313 (1987); B. Martínez and R. Camley, J. Phys., Condens. Matter 4, 5001 (1992).

${ }^{32}$ V. Franco, X. Batlle, A. Labarta, K. O'Grady, and M. L. Watson (unpublished). 